\title{
Systems Medicine - perspectives for collaboration between medical physicists and biomedical engineers
}

\author{
Slavik Tabakov ${ }^{1}$ \\ Received: 30 November 2016 / Accepted: 6 December 2016/Published online: 3 February 2017 \\ (C) IUPESM and Springer-Verlag Berlin Heidelberg 2017
}

Systems Medicine is relatively new interdisciplinary field [1], which looks at the anatomical systems and their functionality as one whole, integrating all their interactions - biochemical, electrophysiological and others, including the environment.

It is known from antiquity that such approach is of extreme importance for the diagnosis and treatment of all human pathological conditions. This has been the basis of medicine for centuries, but now the approach also includes genetic investigations and related bioinformatics and mathematical modelling. This may allow taking into consideration for the diagnosis and treatment not only the statistical parameters of a population, but also the specific physiology of the individual patient, thus creating his unique personal model, predicting the progression of a disease and its reaction to specific medication. This will allow to have unique treatment (and even mathematical testing of individual effect of specific medications) what is the aim of personalized medicine.

Mathematical modelling is one of the subjects of Medical Physics and Biomedical Engineering. This way Systems Medicine development will benefit from close collaboration of medical physicists and biomedical engineers with specialists in biology, bioinformatics and specific fields of medicine.

The International Organization for Medical Physics (IOMP, formed in 1963) is the world's premier international professional organization for medical physics with about 24,000 members in 86 countries. IOMP is charged with a mission to advance medical physics practice worldwide by

This article is part of the Topical Collection on Systems Medicine

Slavik Tabakov

slavik.tabakov@kcl.ac.uk

1 Department Medical Engineering and Physics, King's College London, London, UK disseminating scientific and technical information, fostering the educational and professional development of medical physics and promoting the highest quality medical services for patients [2].

The main activities of medical physicists in the fields of Medical Imaging, Radiotherapy, Radiation Safety, Physiological measurements and others, already contain a number of elements associated with Systems Medicine - for example [3, 4]. This includes various types of Physiological modelling and work in e-Healthcare delivery, while in the Imaging-related research medical physicists are in the forefront of Personalized medicine associated with imaging sciences. A number of projects and publications in the field of Radiobiology modelling are already dealing with issues of System Medicine - such as mathematical models in Radiotherapy, Imaging, Radiation Safety and other fields.

Mathematical modelling is also very strong in the field of Biomedical Engineering - in areas such as electrophysiology and related clinical measurements. The whole process of development and manufacturing of medical devices includes also elements of modelling. The International Federation of Medical and Biological Engineering (IFMBE, formed in 1959) actively support these activities [5], which form some of the main scientific topics in all World Congresses on Medical Physics and Biomedical Engineering.

These activities of both sister professions create excellent background for joint scientific project developments. This can be easily supported by their umbrella organization - the International Union for Physical and Engineering Sciences in Medicine (IUPESM). This Union was formed in 1980 with the specific aim to support the official recognition of both medical physicists and biomedical engineers as unique professional occupations [6]. In achieving its main goal, IUPESM was very successful. It included the professions in the International Council of Scientific Unions (ICSU) during 
1999, and also included the professions in the International Standard Classification of Occupations (ISCO-08), managed by the International Labour Organisation (ILO). This recognition was announced in 2011 [7].

During all this time IUPESM also supported joint scientific collaboration between medical physicists and biomedical engineers, especially during the World Congress of Medical Physics and Biomedical Engineering. With the advancement of System Medicine, new opportunities open for further scientific collaboration between the two professions.

This collaboration will be important for the healthcare delivery of the future, and thus has to be properly planned. A possible way forward is for IUPESM Education and Training Committee to get in touch with the respective Committees of IOMP and IFMBE in order to formulate similar requirements for curriculum (for medical physicists and for biomedical engineers) related to modelling and its mathematical background. This would create a solid base for future cooperation in the field. Alongside this it would be beneficial for both professions to agree on inclusion in their core curricula information (or dedicated modules) which will introduce students to the main elements of System Medicine.

In fact some developed countries already include in their educational systems tools and elements which could be applied in future Systems Medicine research. Usually this is achieved through delivery of specific inter-linked BSc and MSc medical physics courses. Most current tasks of IOMP are related to expanding the main medical physics activities in low-and-middle-income countries, and the coming "Way Forward" IOMP document will address the above discussed professional expansion and inter-professional collaboration.

In parallel, the Scientific Committees of IOMP and IFMBE could plan some joint activities related to specific modelling. This would require also forming links with other biological, medical, bioinformatics and similar scientific societies, as well as Groups and Project he teams working in the area. A possible vehicle for this can be the newly formed Bio-cluster of ICSU [8].

Such a large task could start with emphasis on elements of Systems Medicine, which are already applied by medical physicists - e.g. modelling. An IOMP action plan on the subject could include various activities, such as:

Run Webinars and Overview papers on the subject

Increase the emphasis on modelling in future Scientific Conferences

Develop joint sessions with IFMBE and other parties on the subject
Include topics on personalized medicine at various fora

Include e-Health systems in the training tasks

Encourage inclusion of specific mathematical tools in the education

Encourage inclusion of modelling in educational and training systems

This is a long term plan that could be triggered at the World Congress 2018, but initial steps and preparation can start before this.

The IUPESM Journal Health and Technology [9] is very well placed to support these activities through a sequel of focused issues, addressing Systems Medicine elements. This particular issue, triggered after the very successful MEDICON in Paphos, Cyprus (31 March - 2 April 2016), sets well the trend of the task [10].

While so far the collaboration between medical physicists and biomedical engineers has been mainly in the field of focused research projects, we need to think of further expansion of the scope of the professions in order to prepare better for the challenges of the future. System Medicine has all elements necessary for such expansion. This will help to increase the application of our professional potential for the benefit of the patient and the overall healthcare provision.

\section{Compliance with ethical standards}

Conflict of interest The authors declare that they have no conflict of interest.

\section{References}

1. Kamada T. System biomedicine: a new paradigm in biomedical engineering. Front Med Biol Eng. 1992;4(1):1-2.

2. IOMP official web site - www.iomp.org

3. Chapman JD, Nahum A. Radiotherapy treatment planning: linearquadratic radiobiology. CRC Press; 2015. ISBN 9781439862599.

4. Sitek, A. Statistical computing in nuclear imaging. CRC Press; 2014. ISBN 9781439849347.

5. IFMBE official web site - www.ifmbe.org

6. IUPESM official web site $=$ www.iupesm.org

7. Niroomand-Rad A, Orton C, Smith P, Tabakov S. A history of the International Organization for Medical Physics - 50 years anniversary - part II. J Medical Physics International. 2014;2:7-18. available free from www.mpijournal.org

8. ICSU Biocluster web site - http://www.scoop.it/t/icsu-biocluster

9. IUPESM Journal Health and Technology web site - Springer http://link.springer.com/journal/12553

10. MEDICON 2016 official web site - http://www.cyprusconferences. org/medicon2016/ 